\title{
On the Design of Control Systems over Unreliable Channels
}

\author{
Eduardo I. Silva, Graham C. Goodwin and Daniel E. Quevedo
}

\begin{abstract}
This paper studies LTI one-degree-of-freedom networked control architectures built around LTI SISO plants. The feedback path comprises an unreliable channel that drops data in an i.i.d. fashion. We extend known results to show that there exists a second order moments equivalence between the situation examined and an auxiliary feedback loop where the unreliable channel has been replaced by an additive i.i.d. noise channel that has an equality signal-to-noise ratio constraint. We then use these insights to design optimal controllers. We also establish a necessary and sufficient condition on the successful transmission probability that allows for the design of a controller that guarantees MSS in the considered setup. Our proposal does not require packet arrival acknowledgements. Thus, our results give necessary and sufficient conditions for MSS in an outputfeedback control architecture with UDP-like protocols.
\end{abstract}

\section{INTRODUCTION}

Practical control systems are often subject to communication constraints [1]. Control systems where this happens are called networked control systems (NCSs). In the NCS framework, key questions relate to the interplay between communication constraints and the performance and stability of the resulting closed loop. Key communication constraints are bit-rate limitations, random delays and data-dropouts. A framework for the unified treatment of all these issues does not yet exists, but substantial progress has been made in the study of subproblems. A good overview of control problems subject to data-rate limitations is provided in [2], methodologies to deal with random delays are surveyed in [3], whereas data-loss is studied in, e.g., [4]-[6].

This paper focuses on NCSs where data-dropouts are the only source of communication constraints. In the simplest model of such a situation, uncorrupted data is received at the receiving end with a given probability that is assumed constant over time [4], [5], [7]. Other cases have been treated in, e.g., [8]-[10]. Early work in this area includes [11] and [12], where elementary dropout compensation schemes were studied. The actual design of dropout compensators has been addressed in [13]. The key conclusion in [13] is that, for a specific NCS architecture subject to data-dropouts, the resulting switched system (more precisely, a Markov jump linear system (MJLS)) is equivalent to a linear system with an external noise source having a variance that is proportional to the variance of another signal within the loop. This result is then used to design the dropout compensator so as to minimize the plant output variance.

There exist situations where the performance gains arising from dropout compensation may be not sufficient. In these

E.I. Silva is with the Departamento de Electrónica, UTFSM, Valparaíso, Chile. G.C. Goodwin and D.E. Quevedo are with the School of Electrical Engineering and Computer Science, The University of Newcastle, NSW 2308, Australia. E-mail: eduardo.silva@usm.cl cases, a complete controller re-design is needed. Such an approach has been followed in, e.g., [5], where MJLS theory has been used to synthesize controllers that minimize an $\mathcal{H}_{\infty}$ functional in the presence of i.i.d. data-dropouts. Another relevant work is [7]. In that work, the author studies a general MIMO control problem where communication takes place over a fading network. Section 8 in [7] particularizes the results to a case that employs a single unreliable channel that drops data in an i.i.d. fashion. In this case, and assuming a single input plant whose state is noiselessly measured, it is shown in [7] that an LTI controller that achieves mean square stability (MSS) can be found, if and only if the probability of successful transmission is greater than a function of the product of the unstable plant poles. However, no performance questions are addressed in [7].

Control over unreliable channels has also been addressed from the perspective of classic LQG theory in, e.g., [14] and [4]. The work [14] was one of the first ones to point out that there exist control problems where fundamental differences arise depending on whether or not there exist acknowledgements that testify successful transmissions. If such acknowledgements are available (TCP-like protocols), then the classical separation principle holds, whilst no separation holds when no acknowledgements are available (UDPlike protocols) [4]. It has also been shown in [14] and [4] that, consistent with the results in [7], there exists a region in the plane of successful transmission probabilities within which MSS can be guaranteed. As expected, these regions are protocol dependant. Another conclusion in [4] is that LTI control policies are suboptimal when dealing with datadropouts. Indeed, in the UDP-like protocol case, optimal control laws are non-linear in general and they do not seem to admit an usable characterization [4]. This motivated the study of optimal LTI controllers pursued in [15]. Related results can be found in [6], [16]. In that work, a specific coding and control architecture is proposed (which is more general than those considered in [4], [14], [15]) and it is shown to be optimal irrespective of the actual channel dropout profile.

In this paper we consider a standard LTI one-degree-offreedom (one-dof) architecture built around an LTI SISO plant, where the feedback path comprises an unreliable channel that drops data in an i.i.d. fashion. We adapt the results in [13] to this architecture and show that there exists a second order moments equivalence between the considered NCS and an auxiliary control system where the unreliable channel has been replaced by an additive i.i.d. noise channel that has an equality signal-to-noise ratio (SNR) constraint. We then use these insights to obtain optimal controller designs, and also to establish necessary and sufficient conditions for the 
existence of an LTI controller that achieves MSS.

The results in this paper go beyond the results in [13], where no detailed study of the interplay between MSS and dropout probability is performed. We also extend the results in [7], where no noise sources or disturbances are considered, and where only MSS is studied. On the other hand, since our proposal does not use packet arrival acknowledgements, our results give necessary and sufficient conditions for the existence of LTI controllers that achieve MSS in an outputfeedback UDP-like protocol based networked architecture, whereas the available results (see [4], [14]) give only sufficient or necessary conditions depending on the plant structure.

The remainder of this paper is organized as follows: Section II presents the notation used throughout the paper. Section III states the problem of interest. Section IV shows that the considered NCS architecture is equivalent (in the sense indicated above) to a standard LTI feedback loop with an equality SNR constraint. Section V studied conditions that guarantee the existence of controllers that achieve MSS, and Section VI focuses on performance related questions. Section VII presents an example. Section VIII draws conclusions. Due to space constraints, all proofs have been omitted and can be found in [17] or obtained via e-mail from the authors.

\section{NOTATION}

$\mathbb{N}_{0} \triangleq\{0,1, \cdots\}$ and $\mathbb{R}_{0}^{+} \triangleq\{x \in \mathbb{R}: 0 \leq x<\infty\} . \mathcal{P}\{\cdot\}$ denotes probability, $\mathbb{E}\{\cdot\}$ denotes the expectation operator, $\operatorname{vec}\{\cdot\}$ denotes the column stacking operator, and $\otimes$ is the Kronecker product [18]. Given any scalar $x,|x|$ denotes its magnitude and $\bar{x}$ its complex conjugate. We use $z$ as both the argument of the z-transform and as the forward shift operator, where the meaning is clear from the context.

In this paper, all transfer functions are in discrete time and real rational. Usually, we omit the argument $z$ and write $A$ instead of $A(z) . \mathcal{R}_{p}$ contains all proper transfer functions, $\mathcal{R}_{s p}$ contains all strictly proper transfer functions and $\mathcal{R} \mathcal{H}_{\infty}$ contains all the stable and proper transfer functions. $\mathcal{R} \mathcal{L}_{2}$ contains all real rational discrete time transfer functions with no poles on the unit circle. For any $A \in \mathcal{R} \mathcal{L}_{2}$, we define its $2-$ norm as usual, and denote it by $\|A\|_{2}^{2}$ [19]. If $A \in$ $\mathcal{R} \mathcal{L}_{2}$, then we may write $A=[A]_{\mathcal{H}_{2}^{\perp}}+[A]_{\mathcal{H}_{2}}$, where $[A]_{\mathcal{H}_{2}^{\perp}}$ contains the strictly unstable, constant and non-causal parts of $A$, and $[A]_{\mathcal{H}_{2}}$ corresponds to the stable and strictly proper part of $A$. Sometimes, we write $\left.\{A\}\right|_{z=0}$ instead of $A(0)$. $A^{\sim}$ is a shorthand for $A\left(z^{-1}\right)^{T}$.

Unless otherwise stated, random processes are always scalar and defined for $k \in \mathbb{N}_{0}$. We abbreviate $\{x(k)\}_{k \in \mathbb{N}_{0}}$, $x(k) \in \mathbb{R}$, by $x$. The variance, at time instant $k$, of a process $x$ is denoted via $\sigma_{x}^{2}(k)$. If $x$ is a random variable, then $\sigma_{x}^{2}$ denotes its variance. We define $\sigma_{x}^{2} \triangleq \lim _{k \rightarrow \infty} \sigma_{x}^{2}(k)$, provided the limit exists. If $x$ is a wide sense stationary (wss) (asymptotically wss) process, then $S_{x}\left(e^{j \omega}\right)$ denotes its (stationary) power spectral density (PSD) and $\Omega_{x}$ denotes any spectral factor of $S_{x}\left(e^{j \omega}\right)$, i.e., $\left|\Omega_{x}\left(e^{j \omega}\right)\right|^{2} \triangleq S_{x}\left(e^{j \omega}\right)$. We say that a random variable (process) is a second order

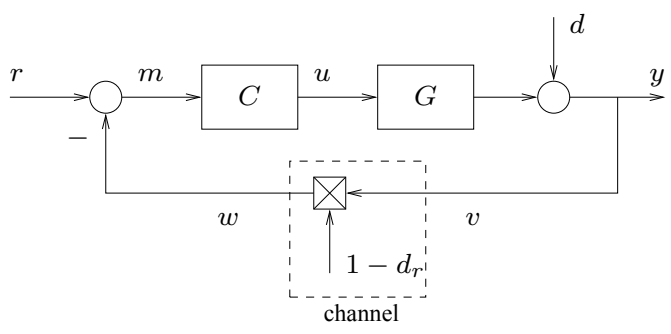

Fig. 1. One degree of freedom control loop with feedback over unreliable channel.

one if and only if it has finite mean and finite second order moments.

\section{Problem Definition}

This paper focuses on a NCS architecture where an LTI one-dof controller has to be designed to optimize performance in a closed loop that uses an unreliable channel in the feedback path. The situation of interest is depicted in Figure 1, where $G$ is the plant transfer function, $C$ is the controller, $r$ is a reference signal, $d$ models plant output disturbances, $y$ is the plant output, and the channel is an analog erasure channel. That is, the channel input $v$ and the channel output $w$ are related via

$$
w(k) \triangleq\left(1-d_{r}(k)\right) v(k), \quad \forall k \in \mathbb{N}_{0}, \quad \forall v(k) \in \mathbb{R},
$$

where $d_{r}$ is a process that models data dropouts and that is such that $d_{r}(k) \in\{0,1\} \forall k \in \mathbb{N}_{0}$.

\section{Assumption 1:}

(a) $d_{r}$ is an independent sequence of i.i.d. Bernoulli random variables such that $\mathcal{P}\left\{d_{r}(k)=1\right\}=1-p, \forall k \in \mathbb{N}_{0}$.

(b) $G \in \mathcal{R}_{s p}, G$ is SISO, non-zero, has no zeros or poles on the unit circle, and has a minimal underlying realization.

(c) $r$ and $d$ are second order jointly independent and mutually uncorrelated wss scalar processes with rational PSDs. At least one signal, $r$ or $d$, is non-zero. If $r$ or $d$ are non-zero, then they admit rational, stable, biproper and minimum-phase spectral factors.

(d) The initial states of both the plant and controller are jointly second order random variables.

According to Assumption 1, the channel in Figure 1 drops data in an i.i.d. fashion. More general channel models have been considered in, e.g., [8]. Assuming that the plant is strictly proper guarantees that the feedback loop of Figure 1 is well-posed for all causal controllers. We focus on SISO plants for simplicity. The remaining assumptions on $G$ are either standard or non-essential to our results (see [20] for more general cases). The assumptions on $r$ and $d$ are standard, except for the fact that we do not allow both $r$ and $d$ to be zero signals for brevity. Finally, the assumptions on the plant and controller initial states are also standard.

In this paper we will use the following notion of stability (see also, e.g., [7], [21]):

Definition 1 (Mean square stability): Consider a system described by $x(k+1)=f(x(k), w(k))$, where $k \in \mathbb{N}_{0}, f$ : $\mathbb{R}^{n} \times \mathbb{R}^{m} \rightarrow \mathbb{R}^{n}, x(k) \in \mathbb{R}^{n}$ is the system state at time instant 


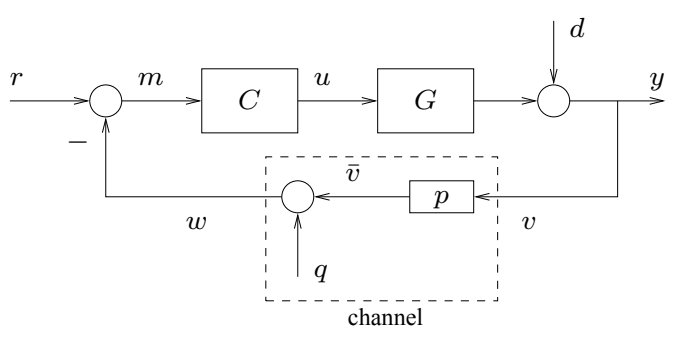

Fig. 2. LTI system with feedback over additive i.i.d. noise channel, plus a gain.

$k, x(0)=x_{o}$, where $x_{o}$ is a second order random variable, and the input $w$ is a second order wss process independent of $x_{o}$. We say that the system is mean square stable (MSS) if and only if there exist finite $\mu \in \mathbb{R}^{n}$ and finite $M \in \mathbb{R}^{n \times n}$, $M \geq 0$, such that

$$
\lim _{k \rightarrow \infty} \mathbb{E}\{x(k)\}=\mu, \quad \lim _{k \rightarrow \infty} \mathbb{E}\left\{x(k) x(k)^{T}\right\}=M,
$$

regardless of the initial state $x_{o}$.

We note that MSS reduces to internal stability (in the standard sense [22]) when $f(x, w)=A x+B w$.

We define the tracking error $e$ via

$$
e \triangleq r-y
$$

and use its stationary variance $\sigma_{e}^{2}$ as performance measure.

We are now in a position to state the problem of interest in this paper:

Problem 1: Consider the NCS of Figure 1 and suppose that Assumption 1 holds. For any successful transmission probability $p \in(0,1)$, find (or prove the problems unfeasible)

$$
\left[\sigma_{e}^{2}\right]_{p} \triangleq \inf _{C \in \mathcal{S}_{p}} \sigma_{e}^{2}, \quad C_{p} \triangleq \arg \inf _{C \in \mathcal{S}_{p}} \sigma_{e}^{2} .
$$

where $\mathcal{S}_{p} \triangleq\left\{C \in \mathcal{R}_{p}\right.$ : the loop of Figure 1 is MSS $\}$.

In Problem 1, we left the extreme cases $p \in\{0,1\}$ aside. These can be studied using standard control theory.

The remainder of this paper focuses on solving Problem 1. To that end, we will first extend known results to show that Problem 1 is equivalent to a problem of control system design with an equality SNR constraint. Once this equivalence is revealed, we will give a characterization of the solution to Problem 1.

\section{Preliminary Results}

Consider an auxiliary situation where the analog erasure channel of Figure 1 is replaced by an additive noise channel plus a gain which is equal to the successful transmission probability $p$ (see Figure 2). In Figure 2, $q$ is an exogenous noise source satisfying the following:

Assumption 2 (Additive noise channel): The signal $q$ is an independent sequence of i.i.d. random variables having zero mean and a variance $\sigma_{q}^{2}$ that satisfies

$$
\sigma_{q}^{2}=p(1-p) \sigma_{v}^{2}
$$

provided the stationary variance of $v$ exists and is finite.

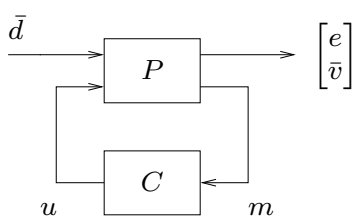

Fig. 3. Equivalent re-writing of Figure 2.

We note that, for $p \in(0,1)$, (1) can be written as

$$
\gamma \triangleq \frac{\sigma_{\bar{v}}^{2}}{\sigma_{q}^{2}}=\frac{p}{1-p}
$$

where $\bar{v} \triangleq p v$ (see Figure 2). The ratio $\gamma$ is the SNR of the additive noise channel in the feedback path of Figure 2.

The next theorem establishes a relationship between the feedback loops of Figures 1 and 2. (This result is implicitly stated in [13] for a different architecture.)

Theorem 1 (Equivalence): Consider the feedback loops of Figures 1 and 2. If $p \in(0,1)$, and Assumptions 1 and 2 hold, then:

1) If the feedback system of Figure 1 is MSS and the feedback system of Figure 2 is internally stable, then the stationary PSDs of $e$ and of all the signals in the loops are the same in both situations.

2) The networked system of Figure 1 is MSS if and only if the feedback loop of Figure 2 is asymptotically stable and

$$
\frac{p}{1-p}>\left\|T_{p}\right\|_{2}^{2}
$$

where $T_{p}$ is the transfer function from $q$ to $\bar{v}$ in Figure 2, namely

$$
T_{p} \triangleq(1-p G C)^{-1} p G C .
$$

Moreover, (3) is equivalent to $\sigma_{q}^{2} \in \mathbb{R}_{0}^{+}$.

We conclude from Theorem 1 that spectral and MSSrelated properties of the NCS of Figure 1 can be rigorously studied using the LTI system of Figure 2, where the unreliable channel has been replaced by an additive i.i.d. noise channel, having gain $p$ and an equality SNR constraint given by (2). This property is central for the remainder of this paper.

We end this section by making explicit a few consequences of Theorem 1. To that end, we write the feedback loop of Figure 2 as in Figure 3, where $P$ is such that

$$
\left[\begin{array}{c}
e \\
\bar{v} \\
\hdashline m
\end{array}\right]=\left[\begin{array}{c:c}
P_{e 11} & P_{e 12} \\
P_{\bar{v} 11} & P_{\bar{v} 12} \\
\hdashline P_{21} & P_{22}
\end{array}\right]\left[\begin{array}{c}
\bar{d} \\
\hdashline u
\end{array}\right],
$$

$\bar{d} \triangleq\left[\begin{array}{lll}r & d & q\end{array}\right]$, and

$$
\begin{array}{rlrl}
P_{e 11} \triangleq\left[\begin{array}{lll}
1 & -1 & 0
\end{array}\right], & & P_{e 12} \triangleq-G, \\
P_{\bar{v} 11} \triangleq\left[\begin{array}{lll}
0 & p & 0
\end{array}\right], & P_{\bar{v} 12} \triangleq p G, \\
P_{21} \triangleq\left[\begin{array}{lll}
1 & -p & -1
\end{array}\right], & P_{22} \triangleq-p G .
\end{array}
$$

Corollary 1: Consider the NCS of Figure 1, assume that $p \in(0,1)$ and that Assumption 1 holds. Recall the definitions 
in (4) and choose $X, Y, N$ and $D$ in $\mathcal{R H}_{\infty}$ such that $X$ and $D$ are biproper, $P_{22}=N D^{-1}$ and $X D-Y N=1$. Then:

1) $C \in \mathcal{S}_{p}$ if and only if $\frac{p}{1-p}>\left\|T_{p}\right\|_{2}^{2}$ and

$$
C=(X-Q N)^{-1}(Y-Q D),
$$

where $Q \in \mathcal{R H}_{\infty}$ is a free parameter.

2) If $C \in \mathcal{S}_{p}$, then the stationary variances $\sigma_{e}^{2}$ and $\sigma_{\bar{v}}^{2}$ exist, are finite and satisfy

$$
\sigma_{e}^{2}=\left\|T_{\bar{d} e} \Omega_{\bar{d}}\right\|_{2}^{2}, \quad \sigma_{\bar{v}}^{2}=\left\|T_{\bar{d} \bar{v}} \Omega_{\bar{d}}\right\|_{2}^{2},
$$

where

$$
\begin{aligned}
& T_{\bar{d} e} \triangleq T_{\overline{d e}}^{o}-P_{e 12} D Q D P_{21}, \\
& T_{\bar{d} \bar{v}} \triangleq T_{\bar{d} \bar{v}}^{o}-P_{\bar{v} 12} D Q D P_{21}, \\
& T_{\overline{d e}}^{o} \triangleq P_{e 11}+P_{e 12} D Y P_{21}, \\
& T_{\bar{d} \bar{v}}^{o} \triangleq P_{\bar{v} 11}+P_{\bar{v} 12} D Y P_{21},
\end{aligned}
$$

and where $\Omega_{\bar{d}} \triangleq \operatorname{diag}\left\{\Omega_{r}, \Omega_{d}, \sigma_{q}\right\}$,

$$
\sigma_{q}^{2}=\frac{\left\|T_{r d \bar{v}} \operatorname{diag}\left\{\Omega_{r}, \Omega_{d}\right\}\right\|_{2}^{2}}{\frac{p}{1-p}-\left\|T_{p}\right\|_{2}^{2}}
$$

and $T_{r d \bar{v}}$ denotes the transfer function from $\left[\begin{array}{ll}r & d\end{array}\right]^{T}$ to $\bar{v}$ in Figure 2

Remark 1: We note that the left hand side in (7) belongs to $\mathbb{R}_{0}^{+}$whenever $C$ is as in (5), and (3) is satisfied, i.e., whenever the NCS of Figure 1 is MSS.

The expressions for $\sigma_{e}^{2}$ and $\sigma_{\bar{v}}^{2}$ provided in (6) are not explicit. Indeed, they have $\sigma_{q}$ as a parameter, which, in turn, can be obtained from (7). Writing $\sigma_{e}^{2}$ and $\sigma_{\bar{v}}^{2}$ as in (6), and keeping in mind that (1) (or (2)) holds, has some advantages that we will exploit below. We also note that, since $G$ is nonzero, both $\sigma_{e}^{2}$ and $\sigma_{\bar{v}}^{2}$ are strictly convex functions of $Q$ for every fixed $\sigma_{q}^{2}$. In the sequel, it will be convenient to make this fact explicit. To that end, we define the functionals

$$
J_{\sigma_{q}^{2}}(Q) \triangleq \sigma_{e}^{2}, \quad R_{\sigma_{q}^{2}}(Q) \triangleq \sigma_{\bar{v}}^{2} .
$$

$J_{\sigma_{q}^{2}}(Q)$ and $R_{\sigma_{q}^{2}}(Q)$ correspond to the stationary variances of $e$ and $\bar{v}$ as a function of $Q$ and with $\sigma_{q}^{2}$ as a parameter, respectively.

\section{Mean Square Stability}

Before solving Problem 1, we will address the simpler problem of characterizing the successful transmission probabilities $p$ that guarantee that the set $\mathcal{S}_{p}$ is non-empty. To that end, we define

$$
\Delta_{G} \triangleq \eta+\delta
$$

where (see also Equation (34) in [23])

$$
\begin{aligned}
\eta \triangleq \sum_{i=1}^{n_{c}} \sum_{j=1}^{n_{c}} \frac{\psi_{i} \bar{\psi}_{j}}{\left(c_{i} \bar{c}_{j}-1\right)}, \\
\psi_{i} \triangleq\left(1-\left|c_{i}\right|^{2}\right)\left(\xi_{p}\left(c_{i}\right)-\sum_{j=0}^{m-1} \beta_{j} c_{i}^{-j}\right) \prod_{\substack{j=1 \\
j \neq i}}^{n_{c}} \frac{1-c_{i} \bar{c}_{j}}{c_{i}-c_{j}}, \\
\xi_{p} \triangleq \prod_{i=1}^{n_{p}} \frac{1-z p_{i}}{z-p_{i}},\left.\quad \beta_{k} \triangleq\left\{\frac{1}{k !} \frac{d^{k}}{d z^{k}} \xi_{p}^{-1}\right\}\right|_{z=0}, \\
\delta \triangleq \begin{cases}0 & \text { if } m=1, \\
\sum_{i=1}^{m-1}\left|\beta_{k}\right|^{2} & \text { if } m>1,\end{cases}
\end{aligned}
$$

$m$ is the relative degree of $G$ and $\left\{c_{1}, \cdots, c_{n_{c}}\right\}$ (resp. $\left\{p_{1}, \cdots, p_{n_{p}}\right\}$ ) denotes the set of NMP zeros (resp. unstable poles) of $G$. It follows from the results in [23] that $\Delta_{G} \geq 0$ and that $\Delta_{G}=0$ if and only if the plant has no NMP zeros and has relative degree one.

Theorem 2 (Minimal $p$ for MSS): Consider the NCS of Figure 1 and suppose that Assumption 1 holds. If $G$ is unstable, then there exists a controller $C \in \mathcal{R}_{p}$ such that MSS holds if and only if

$$
p>p_{\text {inf }} \triangleq 1-\frac{1}{\left(\prod_{i=1}^{n_{p}}\left|p_{i}\right|^{2}\right)+\Delta_{G}} .
$$

Remark 2 (Stable plants): If $G$ is stable, then there exists $C \in \mathcal{R}_{p}$ such that the resulting loop is MSS for any $p \in[0,1]$ (just choose $C=0$ ). Thus, $p_{\text {inf }}=0$ in those cases.

Theorem 2 gives an explicit characterization of the minimal successful transmission probability that guarantees the existence of a controller $C$ that renders the NCS of interest MSS. Our result states that, as intuition suggests, plants that have unstable poles of large magnitude require more reliable channels in order to be stabilized than those plants having unstable poles of moderate size. It is also appreciated that, for the considered control architecture, not only unstable plant poles affect the minimal admissible value for $p$, but also the plant NMP zeros and relative degree.

In the control architecture under study, the sending end does not exploit knowledge of whether or not data arrives uncorrupted at the controller side. As such, the considered scheme can be implemented using UDP-like network protocols. In such cases, the results of Theorem 6.1 in [4] ${ }^{1}$ give necessary conditions on the dropout probabilities that guarantee MSS for the case of plant models that have a square and invertible state-to-output matrix ("C $\mathrm{C}$ " matrix). That condition turns out to be also sufficient in the case of systems that, in addition, have a square and invertible inputto-state matrix ("B" matrix; see also [14]). In the SISO case, having both a square input-to-state and state-to-output matrix is tantamount to having a scalar plant model. Our results are consistent with those reported in [4], [14], but go beyond those results in that they provide necessary and sufficient

\footnotetext{
${ }^{1}$ Note that equation (40) in Theorem 6.1 in [4] contains a typo that is corrected in the proof of that theorem.
} 
conditions that are valid for (almost) any SISO plant model when the control scheme is an LTI one-dof one.

Our results also extend the results in Section 8 in [7]. In that work, the author gives conditions on the successful transmission probability for LTI one-dof control architectures with perfect state feedback. It turns out that, in that special case, (9) with $\Delta_{G}=0$ is necessary and sufficient to be able to find a state feedback law that guarantees MSS. Our results extend the framework in Section 8 of [7] to the output feedback case and show that, in this case, not only the unstable plant poles limit the channel reliability needed to achieve MSS.

\section{DESIGN FOr Performance}

In this section we solve Problem 1. To that end, we use the results of Theorem 1 and focus on the LTI system of Figure 2, where $q$ satisfies Assumption 2. Provided the assumptions of Problem 1 are satisfied, Theorem 1 and Corollary 1 allow one to conclude that $\left[\sigma_{e}^{2}\right]_{p}$ can be written as

$$
\left[\sigma_{e}^{2}\right]_{p}=\inf _{\substack{Q \in \mathcal{R} \mathcal{H}_{\infty} \\ \sigma_{q}^{2} \in \mathbb{R}_{0}^{+} \\ \gamma=\frac{p}{1-p}}} \sigma_{e}^{2},
$$

where $\gamma$ is defined in (2). The first two constraints in the optimization problem in (10) originate from MSS requirements. The third constraint, namely an equality SNR constraint, is a consequence of the fact that the LTI system of Figure 2 models the networked situation of Figure 1 if and only if (2) is satisfied.

We first note that (10) can be written in the equivalent form

$$
\left[\sigma_{e}^{2}\right]_{p}=\inf _{\sigma_{q}^{2} \in \mathbb{R}_{0}^{+}} \inf _{\substack{Q \in \mathcal{R} \mathcal{H}_{\infty} \\ R_{\sigma_{q}^{2}}(Q)=\frac{p}{1-p} \sigma_{q}^{2}}} J_{\sigma_{q}^{2}}(Q),
$$

where $J_{\sigma_{q}^{2}}$ and $R_{\sigma_{q}^{2}}$ are as in (8). For every $\sigma_{q}^{2} \in \mathbb{R}_{0}^{+}$, the inner problem in (11) is a problem of minimizing the variance $\sigma_{e}^{2}$ subject to an equality constraint on the variance $\sigma_{\bar{v}}^{2}$. The Lagrangian associated with this problem is given by

$$
L_{\sigma_{q}^{2}, \epsilon}(Q) \triangleq \epsilon J_{\sigma_{q}^{2}}(Q)+(1-\epsilon) R_{\sigma_{q}^{2}}(Q),
$$

where $\epsilon \in[0,1]$ is a multiplier.

We have the following result:

Lemma 1 (Lagrangian): Consider the NCS of Figure 2, assume that $p \in(0,1]$ and that Assumption 1 holds. Define

$$
\begin{array}{ll}
A_{\epsilon} \triangleq\left[\begin{array}{cl}
\sqrt{\epsilon} T_{\bar{d} e}^{o} \Omega_{\bar{d}} \\
\sqrt{1-\epsilon} T_{\bar{d} \bar{v}}^{o} \Omega_{\bar{d}}
\end{array}\right], & E_{\epsilon} \triangleq\left[\begin{array}{c}
\sqrt{\epsilon} P_{e 12} D \\
\sqrt{1-\epsilon} P_{\bar{v} 12} D
\end{array}\right], \\
A_{v} \triangleq \operatorname{vec}\left\{A_{\epsilon}\right\}, & B_{v} \triangleq\left(D P_{21} \Omega_{\bar{d}}\right)^{T} \otimes E_{\epsilon},
\end{array}
$$

where all symbols are as defined previously. Consider an inner-outer factorization of $B_{v}$ given by $B_{v}=B_{i} B_{o}$, where $B_{i}$ is inner and $B_{o}$ is outer (see, e.g., [19], [24]). Then, for any $\sigma_{q}^{2} \in \mathbb{R}_{0}^{+}$and $\epsilon \in[0,1]$, the parameter $Q$ that minimizes $L_{\sigma_{q}^{2}, \epsilon}($ see (12)) is given by

$$
\begin{aligned}
Q_{\sigma_{q}^{2}, \epsilon} & \triangleq \arg \min _{Q \in \mathcal{R} \mathcal{H}_{\infty}} L_{\sigma_{q}^{2}, \epsilon}(Q) \\
& =B_{o}^{-1}\left(\left.\left\{\left[B_{i}^{\sim} A_{v}\right]_{\mathcal{H}_{2}^{\perp}}\right\}\right|_{z=0}+\left[B_{i}^{\sim} A_{v}\right]_{\mathcal{H}_{2}}\right)
\end{aligned}
$$

The crucial point in calculating $Q_{\sigma_{\alpha}^{2}, \epsilon}$ is the inner-outer factorization of $B_{v}$. This can be done with the aid of the algorithms described in, e.g., [19], [24].

We are now ready to state the main result of this section:

Theorem 3 (Optimal performance): Consider the NCS of Figure 1, suppose that Assumption 1 holds and that $p \in$ $\left(p_{\text {inf }}, 1\right)$. Define the set

$$
\Sigma \triangleq\left\{\sigma_{q}^{2} \in \mathbb{R}_{0}^{+}: R_{\sigma_{q}^{2}}\left(Q_{\sigma_{q}^{2}, 0}\right) \leq \sigma_{q}^{2} \frac{p}{1-p} \leq R_{\sigma_{q}^{2}}\left(Q_{\sigma_{q}^{2}, 1}\right)\right\},
$$

and the function $f: \Sigma \rightarrow[0,1]$ implicitly defined via

$$
\frac{p}{1-p} \sigma_{q}^{2}=R_{\sigma_{q}^{2}}\left(Q_{\sigma_{q}^{2}, f\left(\sigma_{q}^{2}\right)}\right) \text {. }
$$

Then:

1) The minimal stationary tracking error variance $\left[\sigma_{e}^{2}\right]_{p}$ is given by

$$
\left[\sigma_{e}^{2}\right]_{p}=J_{\sigma_{p}^{2}}\left(Q_{\sigma_{p}^{2}, f\left(\sigma_{p}^{2}\right)}\right)
$$

where

$$
\sigma_{p}^{2} \triangleq \arg \min _{\sigma_{q}^{2} \in \Sigma} J_{\sigma_{q}^{2}}\left(Q_{\sigma_{q}^{2}, f\left(\sigma_{q}^{2}\right)}\right) .
$$

2) The optimal controller $C_{p}$ is given by (5) with $Q=$ $Q_{\sigma_{p}^{2}, f\left(\sigma_{p}^{2}\right)}$

Remark 3: We note that Theorem 2 implies that Problem 1 is unfeasible for all $p<p_{\text {inf }}$.

Theorem 3 provides an analytic characterization of the minimal stationary tracking error variance $\sigma_{e}^{2}$ that is achievable with a given successful transmission probability $p$. It also gives the associated optimal controller $C_{p}$. Our results, although analytical, are not explicit since they require the numeric calculation of $\sigma_{p}^{2}$ (see (13)). Nevertheless, they can be used as the basis of simple numerical algorithms to approximate $\left[\sigma_{e}^{2}\right]_{p}$ and $C_{p}$. The following conceptual algorithm illustrates the idea:

Algorithm 1 (Finding $\left[\sigma_{e}^{2}\right]_{p}$ ): For any fixed $p \in(0,1)$ proceed as follows:

- Step 1: Pick a grid $\mathcal{G}=\left\{\sigma_{1}^{2}, \cdots, \sigma_{n}^{2}\right\}$, where $\sigma_{i}^{2} \in \Sigma$ $\forall i \in\{1, \cdots, n\}$.

- Step 2: Find $f\left(\sigma_{i}^{2}\right)$ for every $\sigma_{i}^{2} \in \mathcal{G}$.

- Step 3: Evaluate $\left[\sigma_{e}^{2}\right]_{i} \triangleq J_{\sigma_{i}^{2}}\left(Q_{\sigma_{i}^{2}, f\left(\sigma_{i}^{2}\right)}\right)$ for every $\sigma_{i}^{2} \in$ $\mathcal{G}$. Define $i^{*}$ as the index $i$ associated to the smallest $\left[\sigma_{e}^{2}\right]_{i}$. Approximate $\left[\sigma_{e}^{2}\right]_{p}$ and $Q_{\sigma_{p}^{2}, f\left(\sigma_{p}^{2}\right)}$ by $\left[\sigma_{e}^{2}\right]_{i^{*}}$ and $Q_{\sigma_{i^{*}}^{2}}, f\left(\sigma_{i^{*}}^{2}\right)$, respectively.

We note that $f\left(\sigma_{i}^{2}\right)$ can be found using any line search algorithm. Algorithm 1 can be much improved if the grid $\mathcal{G}$ is iteratively modified, and Steps 2 and 3 are replaced by, e.g., an LMI based optimization procedure that solves the inner problem in (11) and, as a consequence, gives an immediate characterization of $J_{\sigma_{i}^{2}}\left(Q_{\sigma_{i}^{2}, f\left(\sigma_{i}^{2}\right)}\right)$ for every $\sigma_{i}^{2}$ (see, e.g., [25]).

It should be clear that the performance achieved by the controller that our methodology suggests, is only optimal within the class of LTI one-dof control architectures. Better performance may be attained if one considers time varying controllers or more complex architectures as proposed in, 


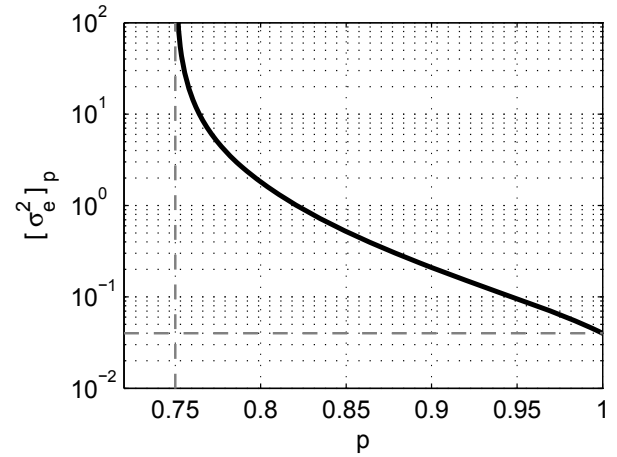

Fig. 4. Best achievable performance as a function of the successful transmission probability $p$ (horizontal line is at $\left[\sigma_{e}^{2}\right]_{p}=D_{\mathrm{inf}}=0.04$ and vertical one is at $p=p_{\text {inf }}=0.75$ ).

e.g., [4], [5]. Indeed, it has been shown in [4] that optimal control policies for control problems that involve unreliable channels are, in general, time varying and, in the case of employing UDP-like protocols (as in our case), non-linear. Our approach, although suboptimal, may allow one to attain acceptable performance levels with a simple LTI architecture.

The work [15] also provides characterizations of LTI controllers for networked situations that are subject to data loss. Our results, as opposed to those in [15], are expressed in simple terms and rely only on standard optimization ideas.

\section{AN EXAMPLE}

This section provides an example to illustrate the results in this paper. Assume that the plant model is given by $G=$ $(z-0.8)(z(z-2))^{-1}$, that $d=0$ and that the reference $r$ has a spectral factor given by $\Omega_{r}=0.1 z(z-0.9)^{-1}$.

The results of Section $\mathrm{V}$ imply that one will be able to find a controller $C$ that renders the networked control loop MSS if and only if $p>p_{\text {inf }}=0.75$. We will thus focus on successful transmission probabilities $p \in(0.75,1]$. Using Theorem 3 and Algorithm 1, we calculated the best achievable performance for such values of $p$. The results are presented in Figure 4. As expected, the best achievable performance is lower bounded by the best non-networked performance, i.e., by

$$
D_{\mathrm{inf}} \triangleq \inf _{Q \in \mathcal{R} \mathcal{H}_{\infty}} J_{0}(Q)=0.04
$$

for every $p$. We also see that $\sigma_{e}^{2} \rightarrow \infty$ when $p \rightarrow p_{\text {inf }}=$ 0.75 , and that $\sigma_{e}^{2} \rightarrow D_{\text {inf }}=0.04$ when $p \rightarrow 1$ (see vertical and horizontal lines in Figure 4, respectively). This behavior is consistent with intuition, and can be easily shown to be consistent with our results.

\section{CONCLUSIONS}

This paper has studied one-dof control loops for SISO LTI plants where the feedback path comprises an unreliable channel. Focusing on LTI control architectures and i.i.d. datadropouts, we showed that the analysis and design of such NCSs can be carried out using standard LTI tools, and design procedures tailored to SNR constrained feedback loops. We believe these insights to be of fundamental importance. Future work should focus on extending the ideas of this paper to situations with multiple channels and to the MIMO case. Extensions to situations that use TCP-like protocols have been explored in [20].

\section{REFERENCES}

[1] J. Hespanha, P. Naghshtabrizi, and Y. Xu, "A survey of recent results in networked control systems," Proceedings of the IEEE, vol. 95, no. 1, pp. 138-162, January 2007.

[2] G. Nair, F. Fagnani, S. Zampieri, and R. Evans, "Feedback control under data rate constraints: An overview," Proceedings of the IEEE, vol. 95, no. 1, pp. 108-137, January 2007.

[3] Y. Tipsuwan and M. Chow, "Control methodologies in networked control," Control Engineering Practice, vol. 11, pp. 1099-1111, 2003.

[4] L. Schenato, B. Sinopoli, M. Franceschetti, K. Poolla, and S. Sastry, "Foundations of control and estimation over lossy networks," Proceedings of the IEEE, vol. 95, no. 1, pp. 163 - 187, January 2007.

[5] P. Seiler and R. Sengupta, "An $\mathcal{H}_{\infty}$ approach to networked control," IEEE Transactions on Automatic Control, vol. 50, no. 3, 2005.

[6] V. Gupta, B. Hassibi, and R. Murray, "Optimal LQG control across packet-dropping links," Systems \& Control Letters, vol. 56, no. 6, pp. 439-446, 2007.

[7] N. Elia, "Remote stabilization over fading channels," Systems \& Control Letters, vol. 54, no. 3, pp. 237-249, 2005.

[8] Q. Ling, "Stability and performance of control systems with limited feedback information," Ph.D. dissertation, University of Notre Dame, Notre Dame, USA, 2005.

[9] W. Zhang, M. Branicky, and S. Phillips, "Stability of networked control systems," IEEE Control Systems Magazine, vol. 21, pp. 84-99, February 2001.

[10] A. Hassibi, S. Boyd, and J. How, "Control of asynchronous dynamical systems with rate constraints on events," in Proceedings of the 38th IEEE Conference on Decision and Control, Phoenix, USA, 1999.

[11] J. Nilsson, "Real-time control systems with delays," Ph.D. dissertation, Lund Institute of Technology, Sweden, 1998.

[12] C. Hadjicostis and R. Touri, "Feedback control utilizing packet dropping network links," in Proceedings of the 41st IEEE Conference on Decision and Control, Las Vegas, USA, 2002.

[13] Q. Ling and M. Lemmon, "Power spectral analysis of networked control systems with data dropouts," IEEE Transactions on Automatic Control, vol. 49, no. 6, pp. 955-960, June 2004.

[14] O. Imer, S. Yüksel, and T. Başar, "Optimal control of LTI systems over unreliable communication links," Automatica, vol. 42, pp. 1429 $-1439,2006$

[15] B. Sinopoli, L. Schenato, M. Franceschetti, K. Poolla, and S. Sastry, "Optimal linear LQG control over lossy networks without packet acknowlegements," Asian Journal of Control, vol. 10, no. 1, 2008.

[16] V. Gupta, D. Spanos, B. Hassibi, and R. Murray, "On LQG control across a stochastic packet-dropping link," in Proceedings of the American Control Conference, Portland, USA, 2005.

[17] E. Silva, G. Goodwin, and D. Quevedo, "On the design of control systems over unreliable channels," Dept. of Electronics, UTFSM, Chile, Tech. Rep., 2009 (available at http: //altair.elo.utfsm.cl/public elo).

[18] D. Bernstein, Matrix Mathematics. Princeton University Press, 2005.

[19] B. Francis, A Course on $H_{\infty}$ Control Theory. Springer, 1987.

[20] E. Silva, "A unified framework for the analysis and design of networked control systems," Ph.D. dissertation, School of Electrical Eng. and Comp. Sci., The University of Newcastle, Australia, 2009.

[21] O. Costa and M. Fragoso, "Stability results for discrete-time linear systems with Markovian jumping parameters," Journal of Mathematical Analysis and Applications, vol. 179, pp. 154-178, 1993.

[22] K. Zhou, J. Doyle, and K. Glover, Robust and optimal control. Prentice Hall, 1996.

[23] J. Braslavsky, R. Middleton, and J. Freudenberg, "Feedback stabilization over signal-to-noise ratio constrained channels," IEEE Transactions on Automatic Control, vol. 52, no. 8, pp. 1391-1403, 2007.

[24] C. Oară, "Constructive solutions to spectral and inner-outer factorizations with respect to the disk," Automatica, vol. 41, pp. 1855-1866, 2005.

[25] S. Boyd, L. El Ghaoui, E. Feron, and V. Balakrishnan, Linear Matrix Inequalities in System and Control Theory. SIAM, 1994. 